\title{
The acoustic field on the forehead of echolocating Atlantic bottlenose dolphins (Tursiops truncatus) ${ }^{\text {a) }}$
}

\author{
Whitlow W. L. Au \\ Hawaii Institute of Marine Biology, P.O. Box 1106 Kailua, Hawaii 96734 \\ Dorian S. Houser \\ Biomimetica, 7951 Shantung Drive, Santee, California 92071 \\ James J. Finneran \\ Space and Naval Warfare Systems Center, U.S. Navy Marine Mammal Program, San Diego, California \\ 92151 \\ Wu-Jung Lee \\ MIT-WHOI Joint Program, Woods Hole Oceanographic Institution, MS \#16 266, Woods Hole Road, Woods \\ Hole, Massachusetts 02543 \\ Lois A. Talmadge and Patrick W. Moore \\ Science Applications International Corporation, 4065 Hancock Street, MS: Q1-A, San Diego, California \\ 92110
}

(Received 30 July 2009; revised 24 November 2009; accepted 28 February 2010)

\begin{abstract}
Arrays of up to six broadband suction cup hydrophones were placed on the forehead of two bottlenose dolphins to determine the location where the beam axis emerges and to examine how signals in the acoustic near-field relate to signals in the far-field. Four different array geometries were used; a linear one with hydrophones arranged along the midline of the forehead, and two around the front of the melon at 1.4 and $4.2 \mathrm{~cm}$ above the rostrum insertion, and one across the melon in certain locations not measured by other configurations. The beam axis was found to be close to the midline of the melon, approximately $5.4 \mathrm{~cm}$ above the rostrum insert for both animals. The signal path coincided with the low-density, low-velocity core of the melon; however, the data suggest that the signals are focused mainly by the air sacs. Slight asymmetry in the signals were found with higher amplitudes on the right side of the forehead. Although the signal waveform measured on the melon appeared distorted, when they are mathematically summed in the far-field, taking into account the relative time of arrival of the signals, the resultant waveform matched that measured by the hydrophone located at $1 \mathrm{~m}$.
\end{abstract}

(C) 2010 Acoustical Society of America. [DOI: 10.1121/1.3372643]

PACS number(s): 43.80.Ka, 43.80.Ev [MCH]

Pages: $1426-1434$

\section{INTRODUCTION}

${ }^{1}$ The forehead of a dolphin is a complex structure with the upper jaw bone, air passages, air sacs, connective tissues, and a bulbous melon that is comprised of translucent lipid very rich in oil, and is referred to as "acoustic fat" (Varanasi and Malin, 1971). Only in the melon and lower jaw can this lipid material be found. Wood (1964) was one of the first to suggest that the fatty melon of the dolphin's forehead may be used to couple sounds from inside the animal's head into the water. Varanasi and Malin (1971) were among the first to study the chemical composition of the melon lipid; they found that it was composed mainly of triacylglycerol and wax esters. Since then, a number of studies have been performed on the chemical, acoustical, and mechanical properties of melon lipids for a variety of odontocete species (Blomberg, 1974; Litchfield et al., 1971; Varanasi and Malin, 1971, 1972). The topography of lipid fractions is thought to

\footnotetext{
This work was presented at the 5th Animal Sonar Symposium, Kyoto, Japan, 14-18 September 2009.
}

be at the functional apex of the refractive process in the beam formation (Norris, 1968). Sound velocity measurements of tissue samples from a dolphin melon indicated a graded sound velocity profile, with a low-velocity core near the midline of the melon and increasing velocity outwards toward the surface of the melon (Litchfield et al., 1979; Norris and Harvey, 1974). A low-density core that likely corresponds to the low-velocity pathway through the melon has been shown in X-ray computer tomography (CT) scans (Cranford, 1988; Cranford et al., 1996). Sounds propagating in an inhomogeneous sound velocity structure will be governed by Snell's law so that as sound propagates through a medium of changing sound velocity, the sound will refract or bend toward the lower velocity region (Urick, 1983).

Although the melons of dolphins have a graded sound velocity and density profile, the mathematical modeling by Aroyan et al. (1992) suggest that the skull and air sacs were the dominant factor in shaping the beam. They go on to 
further state that melon could produce only mild focusing and by itself could not be responsible for the dolphin's highly directive beam.

Au (1993) simultaneously measured the echolocation signals on either the melon or rostrum of a bottlenose dolphin (Tursiops truncates) and at $1 \mathrm{~m}$ from the site of the sound generator. They found the signals measured at the melon to consist of the direct and secondary interferences from the clicks reflecting off internal surfaces of the forehead. These interferences did not exist in the data obtained by Au et al. (2006) using four suction cup hydrophones to examine the acoustic field on the forehead of two echolocating harbor porpoises (Phocoena phocoena). However, there are some major differences in the echolocation signals used by harbor porpoise and bottlenose dolphins. The bandwidth of the echolocation signals of Phocoena phocoena are narrower $(\sim 10-15 \mathrm{kHz})$ compared to about $30-60 \mathrm{kHz}$ for Tursiops truncates, and the signals are projected in a wider beam of approximately $16^{\circ}$ compared to $10^{\circ}(3 \mathrm{~dB}$ beamwidth) for Tursiops truncates (Au, 1993). Furthermore, the signals do not undergo much distortion as a function of angle about the porpoise head within $\pm 20^{\circ}$ (Au et al., 1999). For Tursiops truncates the signals were considerable distorted when measured at $\pm 20^{\circ}$ from the beam axis in both the vertical and horizontal planes (Au, 1993). Au et al. (2006) also found that the beam emerged from the porpoise head approximately $5.6-6.1 \mathrm{~cm}$ from the edge of the animal's upper lip along the midline of the melon, and this location coincided with a pathway extending from the phonic lips through the axis of the low-density/low sound velocity lipid core of the melon. Unfortunately, Au et al. (2006) did not place any hydrophones at locations far from the midline of the porpoise "s head where the beam emerged to consider the role of air sacs.

The objectives of this study were to determine the location on the bottlenose dolphin forehead where the beam axis emerges and to determine the relationship between this location and the low-velocity axis of the melon. The role of air sacs in the focusing of the beam will also be considered. The amplitude distribution of the acoustic pressure of echolocation signals on the forehead was also determined along with the relationship of signal waveforms measured on the forehead with signals in the far-field at $1 \mathrm{~m}$ from the tip of the rostrum.

\section{EXPERIMENTAL PROCEDURE}

\section{A. Echolocation task}

The study was conducted at the Space and Naval Warfare Systems facility in San Diego Bay, where bottlenose dolphins are housed in floating pens. The experimental geometry was described by Moore et al. (2008) in which the dolphin, Heptuna, was trained to station on a submerged biteplate, as shown in Fig. 1 to perform an echolocation task. A second dolphin by the name of Bugs was also used in this study. The target was either a $17.4 \mathrm{~cm}$ diameter stainless steel water-filled sphere or a hollow aluminum cylinder with a height of $17.8 \mathrm{~cm}$, outer diameter of $7.6 \mathrm{~cm}$, and a wall thickness of $9.5 \mathrm{~mm}$. After stationing on the bite plate, the

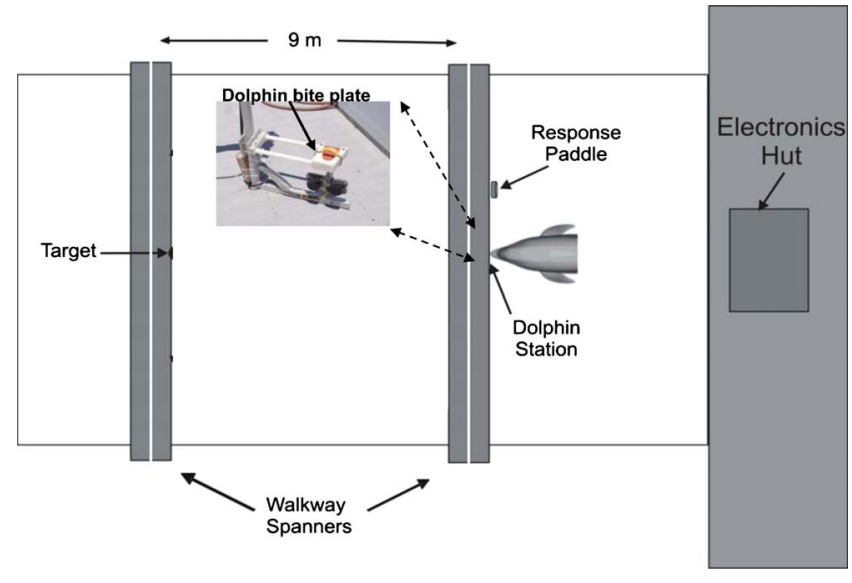

FIG. 1. (Color online) Experimental geometry showing the bite plate station (insert) and the animal stationed on the bite plate facing the target.

subject began echolocating immediately after an acoustically opaque screen $\left(1.1 \times 0.9 \mathrm{~m}^{2}, 12.7 \mathrm{~mm}\right.$ sheet-PVC coated with $6.3 \mathrm{~mm}$ closed cell neoprene) situated $23 \mathrm{~cm}$ beyond the biteplate was raised, using a pulley. A larger sheet of visually opaque polyethylene $(0.4 \mathrm{~mm}$ thick $)$ attached to a waterflooded $1 / 2$ in. PVC pipe frame was fixed directly behind the acoustically opaque screen to prevented visual inspection of the target condition. The animal's position on the bite plate was monitored with a television (TV) camera directly above the bite plate and a TV monitor in the electronic shack. A trial began by placing the suction cup hydrophones in a prearranged pattern on the forehead of the dolphin and having the dolphin station on the bite plate. A go/no-go experimental paradigm was used, in which the subject was trained to back off the bite plate and touch the response paddle to report a target present or go condition. The dolphin had $5 \mathrm{~s}$ from the time the screen was raised to touch the response paddle for a target present response. For a target absent or no-go response, the dolphin had to remain on the bite plate for $5 \mathrm{~s}$ after the target screen was raised. Correct responses were reinforced with a fish reward, and no reinforcement was provided for incorrect reports. An equal number of target present and absent trials was conducted each session. Trials were presented in a random order according to a modification of the Gellerman series (Gellerman, 1933). An equal number of target present and target absent trials was used throughout the experiment. If the suction cups remained firmly on the animal, the next trial would commence with the suction cups left in place. The majority of the trails were conducted with the target directly in front of the animal at a range of $9 \mathrm{~m}$.

\section{B. Hydrophone geometry}

Up to six specially constructed "suction cup" hydrophones were used to measure echolocation signals, a technique first used by Diercks et al. (1973). Four of these hydrophones were the same as the ones used by Au et al. (2006). Each hydrophone consisted of a cylindrical lead zirconate titanate (PZT) piezoelectric element similar to the elements used in the Brüel and Kjaer 8103 hydrophones, but enclosed within a suction cup constructed of degassed polyurethane compound (Uralite 3138). Each piezoelectric cylin- 
(a)

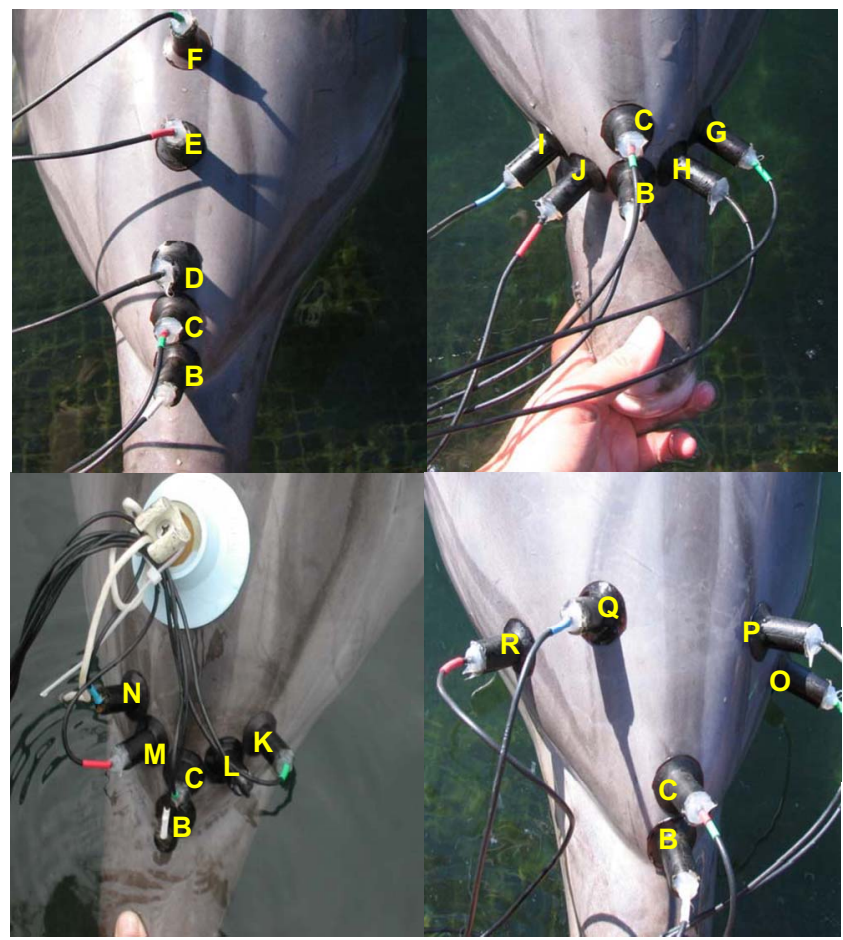

(c)

(d)

FIG. 2. (Color online) Suction cup hydrophone geometry: (a) linear array, (b) curve-1 array, (c) curve-2 array, and (d) curve-3 array. The hydrophone locations are denoted by alphabets.

der had an outer diameter of $6.35 \mathrm{~mm}$, a wall thickness of $1.15 \mathrm{~mm}$, and a height of $6.35 \mathrm{~mm}$. The diameter at the base of a suction cup when attached to the porpoise measured 2.8 $\mathrm{cm}$. A Reson TC4013 was always located directly in front of the animal at a range of $1.2 \mathrm{~m}$ from the assumed source, with the phonic lips just below the anterior portion of the blow hole (Cranford, 2000). The suction cup hydrophones were calibrated in a test tank from 30 to $100 \mathrm{kHz}$, and the individual sensitivity results were used to determine the sound pressure levels of the recorded signals.

Four arrangements of the suction cup hydrophones were used in this project. The first and simplest configuration consisted of five hydrophones arranged as a linear array along the midline of the dolphins' forehead, as shown in Fig. 2(a). The individual hydrophones were identified by different color shrink tubings on the coaxial cable emanating from the suction cups. Hydrophone B with the white shrink tubing was always located on the midline of the forehead with the base of the suction cup at the intersection of the forehead and rostrum, commonly referred to as the rostrum insertion. Hydrophone C was always located next to the "white" suction cup, higher up the forehead with the base of the two suction cups touching each other. Having these two suction cups always at the same locations aided in positioning the other hydrophones relatively consistently from trial to trial and between the two dolphins. Hydrophone B was located with one edge touching the rostrum insertion. The edge of hydrophones $\mathrm{C}$ was in contact with the edge of hydrophone $\mathrm{B}$, and the edge of hydrophone $\mathrm{D}$ touched the edge of hydrophone C. There was an empty spot that was $5.6 \mathrm{~cm}$ (two suction cup width) between hydrophones $\mathrm{D}$ and $\mathrm{E}$, and a similar empty space between hydrophone $\mathrm{E}$ and $\mathrm{F}$. This array configuration was referred to as the linear array. Modified linear-array sessions denoted as linear-R sessions were also conducted, in which a hydrophone was placed on the rostrum instead of location $\mathrm{F}$.

The second hydrophone arrangement consisted of six suction cups wrapped around the forehead with the base of each cup touching the crease at the rostrum insertion as well as each other, as shown in Fig. 2(b). This arrangement was referred to as curve- 1 configuration. The third arrangement of hydrophone consisted of six suction cups wrapped around the forehead but at one suction cup distance $(2.8 \mathrm{~cm})$ above the previous arrangement and touching each other, as shown in Fig. 2(c). This arrangement was referred to as the curve-2 configuration. The fourth arrangement consisted of four of the suction cup hydrophone placed higher up the melon toward the blowhole and spaced evenly about the midline of the melon, as shown in Fig. 2(d). This fourth arrangement was referred to as the curve- 3 configuration. With these four arrangements for the suction cup hydrophones, the amplitude distribution about the forehead of the dolphins could be determined.

Each hydrophone was coupled to an analog in-line filter amplifier Reson VP-1000 set for $20 \mathrm{~dB}$ of gain and with bandpass filter setting from 10 to $150 \mathrm{kHz}$. The clicks recorded on all channels were simultaneously digitized and stored to a personal computer using a 32-channel Interactive Circuits and Systems Limited analog to digital converter using a $312.5 \mathrm{kHz}$ sample rate per channel, 16 bit resolution, and $16 \mathrm{Mbytes} / \mathrm{s}$ transfer rate. Data were collected for a period of $5 \mathrm{~s}$ from the start of each trial, which was sufficient time for the dolphin to echolocate and make a decision regarding the presence of the target.

\section{RESULTS}

Linear array. An example of the waveform and frequency spectrum of the signals measured by each hydrophone in the linear-array geometry for Heptuna is shown in Fig. 3. The hydrophone locations are denoted by alphabets, as shown in Fig. 3(a) for the linear hydrophone geometry. The bottom panel was from the hydrophone $1 \mathrm{~m}$ from the tip of the dolphin rostrum and represents the echolocation in the far-field of the dolphin's head. Note that in the far-field, the peak frequency (frequency at which the energy peaks) was approximately $53 \mathrm{kHz}$ and that none of the signals measured by the hydrophones on the melon had a peak frequency that was close to the far-field peak frequency. The contact hydrophone at location D measured a signal with a peak frequency slightly over an octave higher $(110 \mathrm{kHz})$ than the signal in the far-field and seemed to the result of interference caused by the clicks traveling along different pathways and meeting at location D. The delay of the click traveling along a longer pathway is such that summation caused the peak frequency to shift to a higher value than for the signals measure by the other hydrophones. The amplitude scale for all the signals measured on the forehead was the same so that the differences in the peak-to-peak amplitude can be readily seen. A 


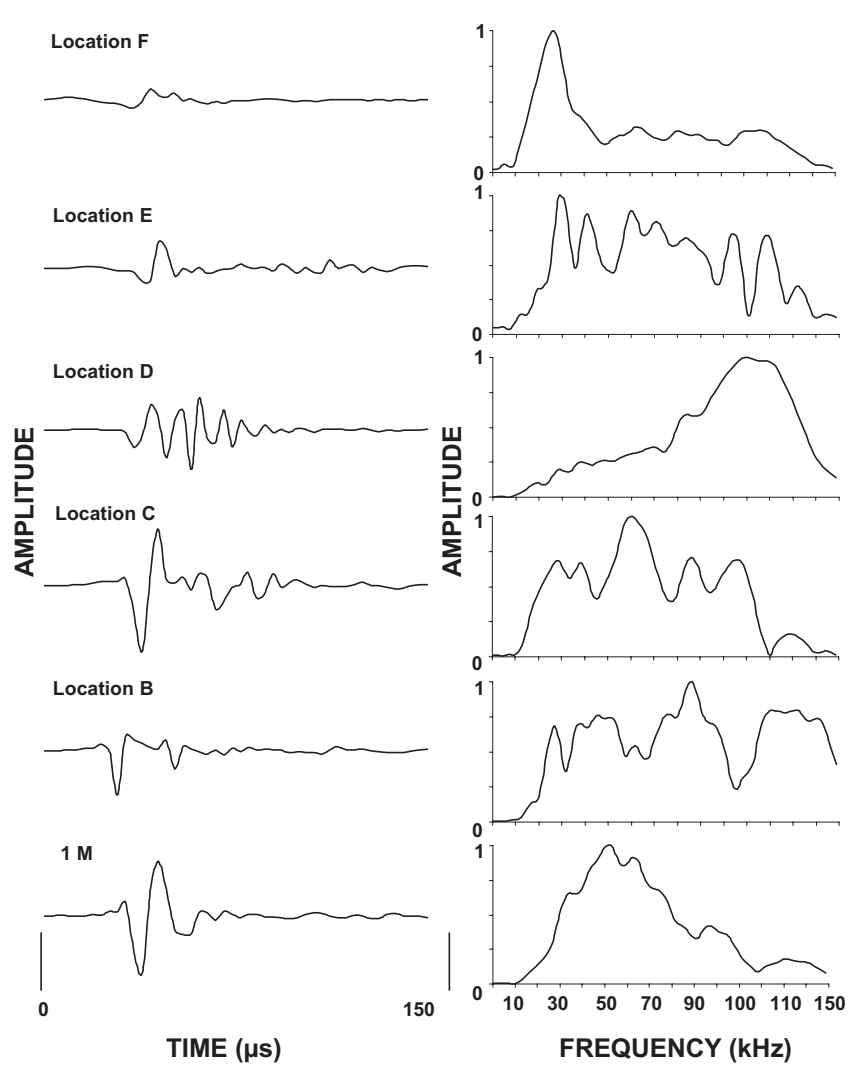

FIG. 3. Examples of a single echolocation click as measured by the hydrophones in the linear-array geometry; the waveforms are shown on the left and spectra are shown on the right.

different scale was used for the signal measured at $1 \mathrm{~m}$, which was usually about 0.3 times lower than the amplitude at location $\mathrm{C}$. This arbitrariness in scaling the amplitude of the signal at $1 \mathrm{~m}$ was done so that the waveform of the signal could be more readily seen with respect to signals measured on the forehead.

The signals in Fig. 3 clearly shows that the signals measured by the different hydrophones were different from each other in both the time and frequency domain. The ripples in the frequency spectra are indicative of multipath propagation, combining the direct signal and reflected signal from internal features within the forehead (Au, 1993). Such multipath propagation was not evident in the harbor porpoise (Au et al., 2006). Unfortunately, we did not compute the frequency spectra for the signals measured by the four hydrophones in the linear geometry in the harbor porpoise measurements. However, the waveforms did not show any evidence of multipath propagation. All the waveforms had very similar shape except for their amplitude. Perhaps the narrowband nature of the harbor porpoise echolocation clicks did not have sufficient resolution to be able to see the effects of multiple arrival at any of the hydrophones.

The mean and standard deviation of peak-to-peak sound pressure levels (SPLs) measured at the various locations on the forehead and at $1 \mathrm{~m}$ from the tip of the rostrum for Heptuna are shown in Fig. 4(a) and in Fig. 4(b) for Bugs. The vertical dashed lines indicate the locations where the two highest levels were measured. For Heptuna [Fig. 4(a)] the means of the amplitudes measured by the hydrophones at

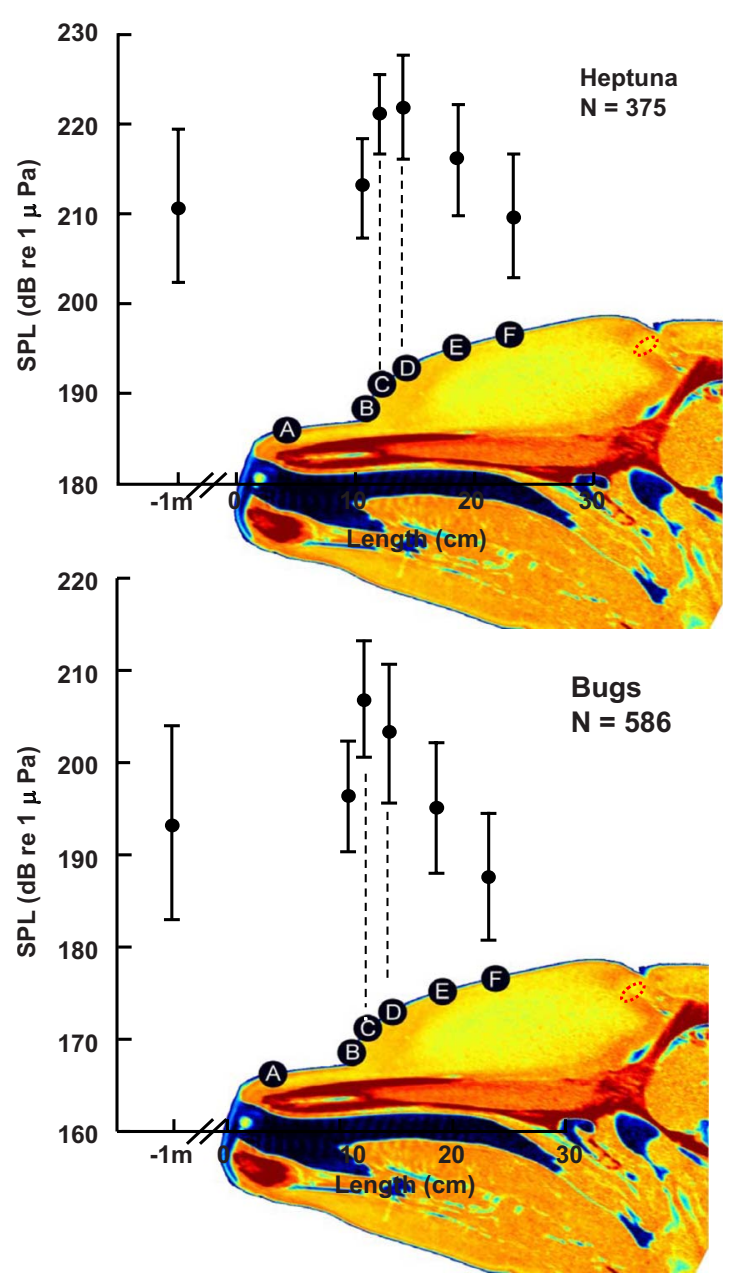

FIG. 4. (Color online) The mean and standard deviation of the peak-to-peak amplitude measured by the different hydrophones in the linear-array geometry and by the hydrophone $1 \mathrm{~m}$ from the anterior tip of the rostrum.

positions $\mathrm{C}$ and $\mathrm{D}$ were not significantly different (2t-test, $p=0.05)$ so that the position where the maximum peak-topeak amplitude can be considered to be somewhere between locations C and D. For Bugs, the maximum amplitude of the echolocation signal was measured by hydrophone $\mathrm{C}$. The mean amplitudes measured by the hydrophones $\mathrm{C}$ and $\mathrm{D}$ were significantly different $(2$ t-test, $p<0.001)$ so that the beam axis can be considered to be close to location $\mathrm{C}$.

In the linear-R sessions [Figs. 5(a) and 5(b)] the maximum SPL was always at location $\mathrm{C}$ for both dolphins; however, the means of the amplitudes measured by the hydrophones at positions $\mathrm{C}$ and $\mathrm{D}$ for Heptuna were not significantly different (2t-test, $p=0.05)$. The maximum SPL for Bugs was also measured by hydrophone $\mathrm{C}$, and mean amplitudes measured at locations $\mathrm{C}$ and D were significantly different (2t-test, $p<0.001)$. The peak-to-peak SPL at the rostrum was $4.5 \mathrm{~dB}$ greater than the SPL at location $\mathrm{C}$ for Heptuna and 4.6 dB for Bugs. This is consistent with the results of Au et al. (1978) in which the hydrophone on the rostrum measured a higher amplitude than when that hydrophone was placed on the melon. However, the pathway to the hydrophone on the rostrum is not obvious. Signals measured at the rostrum had the highest amplitudes for both dolphins. It is difficult to tell whether the signals at the rostrum were 


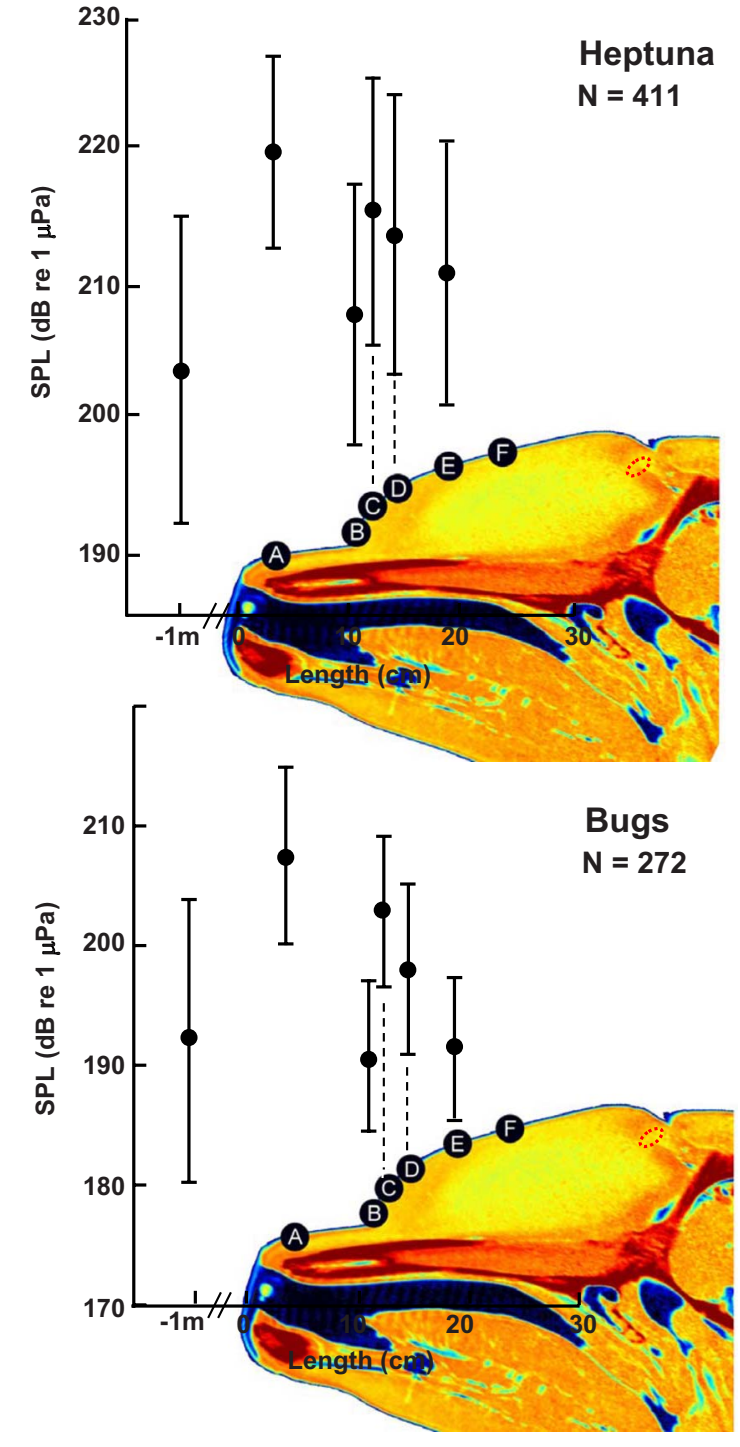

FIG. 5. (Color online) The mean and standard deviation of the peak-to-peak amplitude measured by the different hydrophones in the modified lineararray geometry with one of the suction cup hydrophone on the rostrum and by the hydrophone $1 \mathrm{~m}$ from the anterior tip of the rostrum.

the results of body borne waves or the results of signals radiated out of the forehead and being reinforced at the position of the rostrum. There is also the possibility that the signals at the rostrum were a combination of water-borne and tissue-borne pathways. Unfortunately, it is not obvious why the amplitudes at the rostrum were higher than at any of the other locations either on the forehead or at $1 \mathrm{~m}$.

The results of Figs. 4 and 5 also indicated that Heptuna emitted higher amplitude signals than Bugs by $11-15 \mathrm{~dB}$, as measured by the hydrophone at $1 \mathrm{~m}$, and between 13 and 18 $\mathrm{dB}$ for hydrophone $\mathrm{C}$ on the forehead. In the study of $\mathrm{Au}$ et al. (1978), the hydrophone on the forehead of a bottlenose dolphin measured a peak-to-peak sound pressure level that was approximately $2 \mathrm{~dB}$ lower than at $1 \mathrm{~m}$. The results in this study for the hydrophone at location F (which was approximately at the same location for the measurement of $\mathrm{Au}$ et al., 1978) showed a $1 \mathrm{~dB}$ difference for Heptuna and a 4 $\mathrm{dB}$ difference for Bugs, indicating a consistency between this and the previously study.

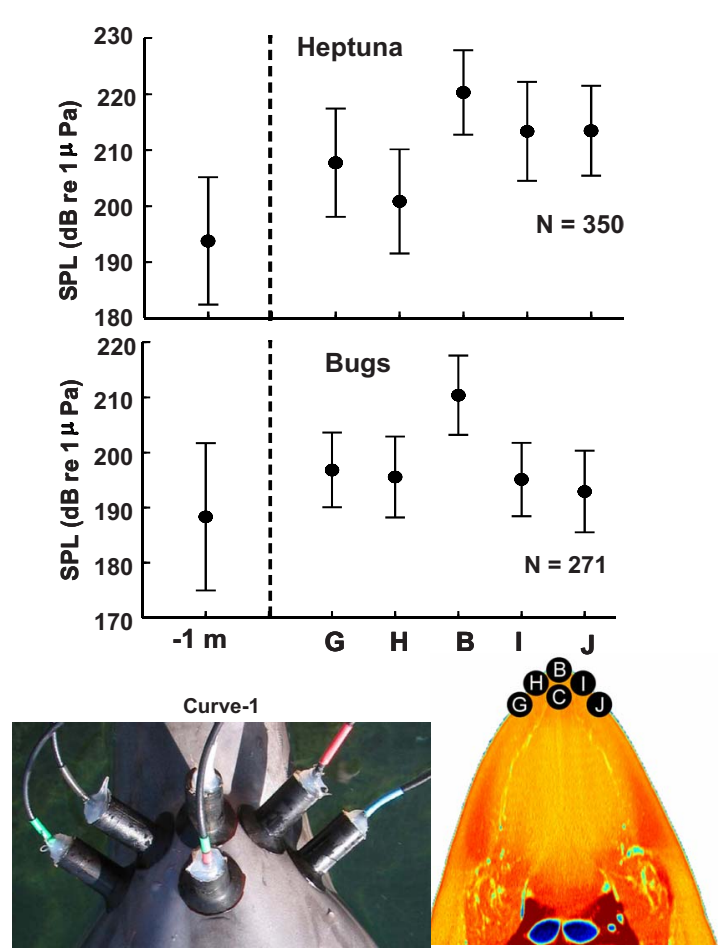

FIG. 6. (Color online) The mean and standard deviation of the peak-to-peak amplitude measured by the hydrophones in the curve- 1 array geometry and the hydrophone at $1 \mathrm{~m}$ from the tip of the animal's rostrum. The position of the hydrophones on the animal's forehead is shown in the bottom left insert. The position of the hydrophones with respect to the horizontal slice of a CT at the approximate level of the suction cup hydrophones. Hydrophone $\mathrm{C}$ is not in the same plane as the other hydrophones.

Curved array. The peak-to-peak SPL measured by the hydrophones in the curve-1 configuration are shown in Fig. 6. The relative positions of the hydrophones are shown on top of the CT scan that consisted of a horizontal slice of the melon at approximately the same plane of the five hydrophones that were position below hydrophone $\mathrm{C}$. The results obtained with the hydrophone at $\mathrm{C}$ were not included in the graph so that the amplitudes along the same plane above the rostrum insertion could be displayed. The hydrophone at B, which was along the midline of the forehead, measured the highest amplitude signals, while the amplitudes measured by the hydrophones off the midline dropped off by approximately $7 \mathrm{~dB}$ on the starboard side for Heptuna and about 12 $\mathrm{dB}$ for the hydrophone at location $\mathrm{G}$ and about $10 \mathrm{~dB}$ for the hydrophone at location $\mathrm{H}$. The drop off for Bugs was nearly the same for all the hydrophones off the midline, approximately $15 \mathrm{~dB}$.

The SPL measured further up the forehead by the hydrophones in the curve- 2 configuration are shown in Fig. 7. Hydrophone $\mathrm{C}$ at the midline measured the maximum values and was consistent with measurements for the curve-1 geometry. There was an asymmetry in the amplitude of the signals measured by the hydrophones located away from the midline, with the amplitude on the right side being about $8 \mathrm{~dB}$ higher than the amplitude of the signal measured on the left side. The shape of the graphs displaying the results for both dolphins was very similar with the SPL on the right side being nearly the same and slightly lower than the SPL at the midline and the SPL on the left side also being nearly the 


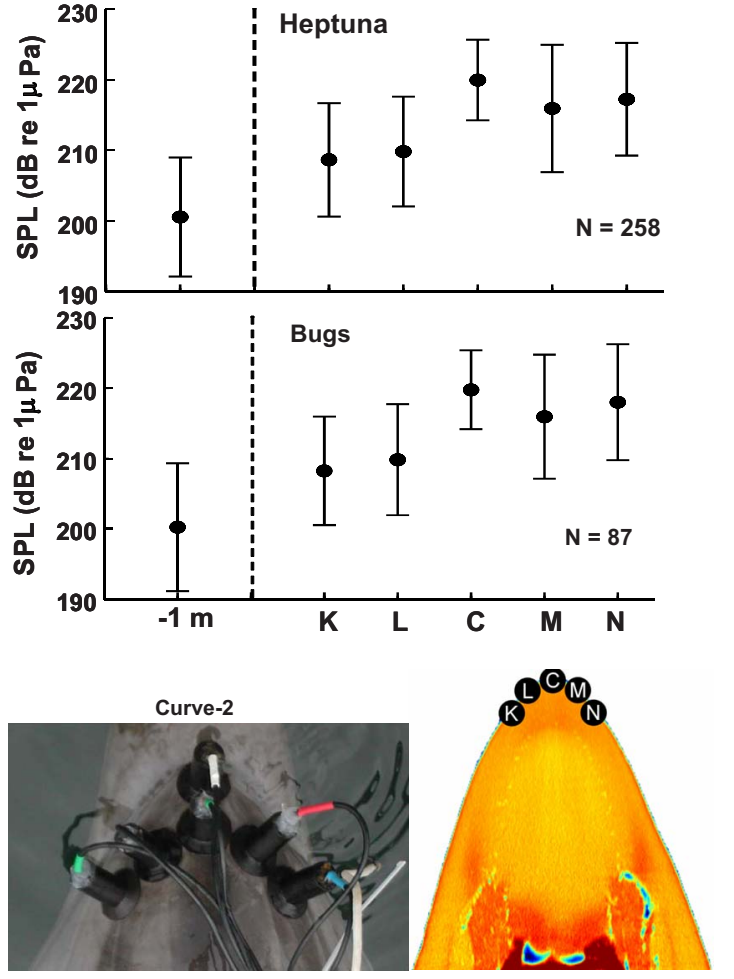

FIG. 7. (Color online) The mean and standard deviation of the peak-to-peak amplitude measured by the hydrophones in the curve- 2 array geometry and the hydrophone at $1 \mathrm{~m}$ from the tip of the animal's rostrum. The position of the hydrophones on the animal's forehead is shown in the bottom left insert. The position of the hydrophones with respect to the horizontal slice of a CT at the approximate level of the suction cup hydrophones.

same but with a greater drop off. The amplitude of Heptuna's signals measured in the curve- 1 and curve- 2 array configurations were relatively similar in value.

Curve 3 array geometry consisted of four hydrophones being located at a considerable distance from the beam axis. This geometry was used to obtain amplitude information away from the beam axis and provide amplitude distribution information over a large section of the forehead. The results are shown in Fig. 8, and as could be expected the hydrophone at the midline of the dolphin forehead recorded the highest amplitude signals with the amplitudes dropping off considerably away from the midline. For Heptuna, the two hydrophones on the right measured amplitudes that were 17 and $25 \mathrm{~dB}$ lower, respectively, than for the hydrophone at location $\mathrm{C}$. The two hydrophones on the left side measured amplitudes that were 23 and $16 \mathrm{~dB}$ lower, respectively, than the hydrophone at location C. For Bugs, the two starboard hydrophones measured amplitudes that were 17 and $21 \mathrm{~dB}$, lower, respectively, than for the hydrophone at location C. The two hydrophones on the left side measured amplitudes that were 27 and $21 \mathrm{~dB}$ lower, respectively, than the hydrophone at location $\mathrm{C}$.

Amplitude distribution. All the results from the various hydrophone geometry can now be used to infer or estimate the typical acoustic amplitude distribution on the forehead of an echolocating dolphin. In order to do this the amplitude from the hydrophone at location $\mathrm{C}$ was chosen as the reference hydrophone, and all the relative amplitude in $\mathrm{dB}$ of the signals measured by each hydrophone was referenced to hy-

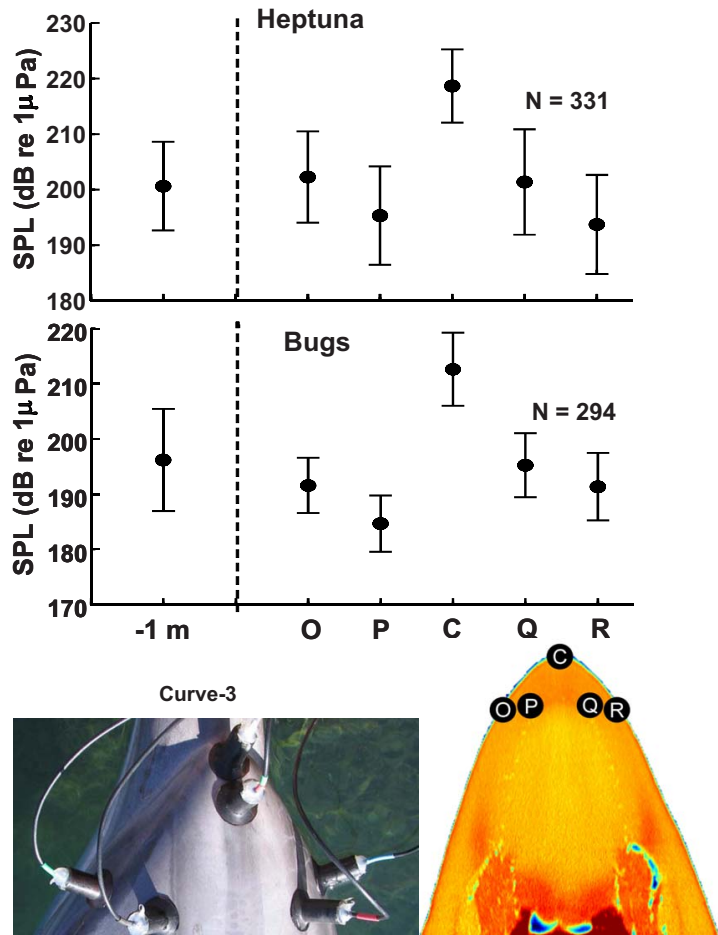

FIG. 8. (Color online) The mean and standard deviation of the peak-to-peak amplitude measured by the hydrophones in the curve- 3 array geometry and the hydrophone at $1 \mathrm{~m}$ from the tip of the animal's rostrum. The position of the hydrophones on the animal's forehead is shown in the bottom left insert. The position of the hydrophones with respect to the horizontal slice of a CT at the approximate level of the suction cup hydrophones.

drophone $\mathrm{C}$ on a click to click basis. Then the means of each of the relative differences were computed. The results of this calculation are shown in Fig. 9 for both dolphins. There is a right side bias to the amplitude distribution that is more obvious from the results obtained with Heptuna. For the purpose of this paper, the area inside of the dashed curve can be considered the area of maximum acoustic amplitude of echolocation signals. The amplitudes on the right side of Heptuna's forehead do not drop off nearly as rapidly as the amplitude on the port side. This right side bias is not as obvious for Bugs since the amplitudes dropped off very rapidly on both sides of the Bugs forehead with distance from the midline. Therefore, the area of higher relative amplitudes $(0-5 \mathrm{~dB})$ is larger for Heptuna in comparison with Bugs. The large differences in the amplitude distribution suggest anatomical differences in both the shape and perhaps the gradient of the sound velocity profile in the melon of both dolphins. It is also important to note that signal amplitudes to the side of the forehead were very low, -17 to $-28 \mathrm{~dB}$ lower than the amplitudes measured by the reference hydrophone. This suggest that sounds may be channeled by reflective processes by the air sacs.

Signal reconstruction. The mean and standard deviation of the time delay in $\mu$ s between the signal received by the hydrophone at location $\mathrm{F}$ and the other hydrophones on the forehead at each location are shown in Fig. 10 for Heptuna and Fig. 11 for Bugs. The time delays associated with locations for the hydrophones at locations $\mathrm{C}, \mathrm{D}$, and $\mathrm{E}$ are very similar between the two animals, being at most only $2 \mu$ difference. However, at location B, there was a $6 \mu$ s differ- 

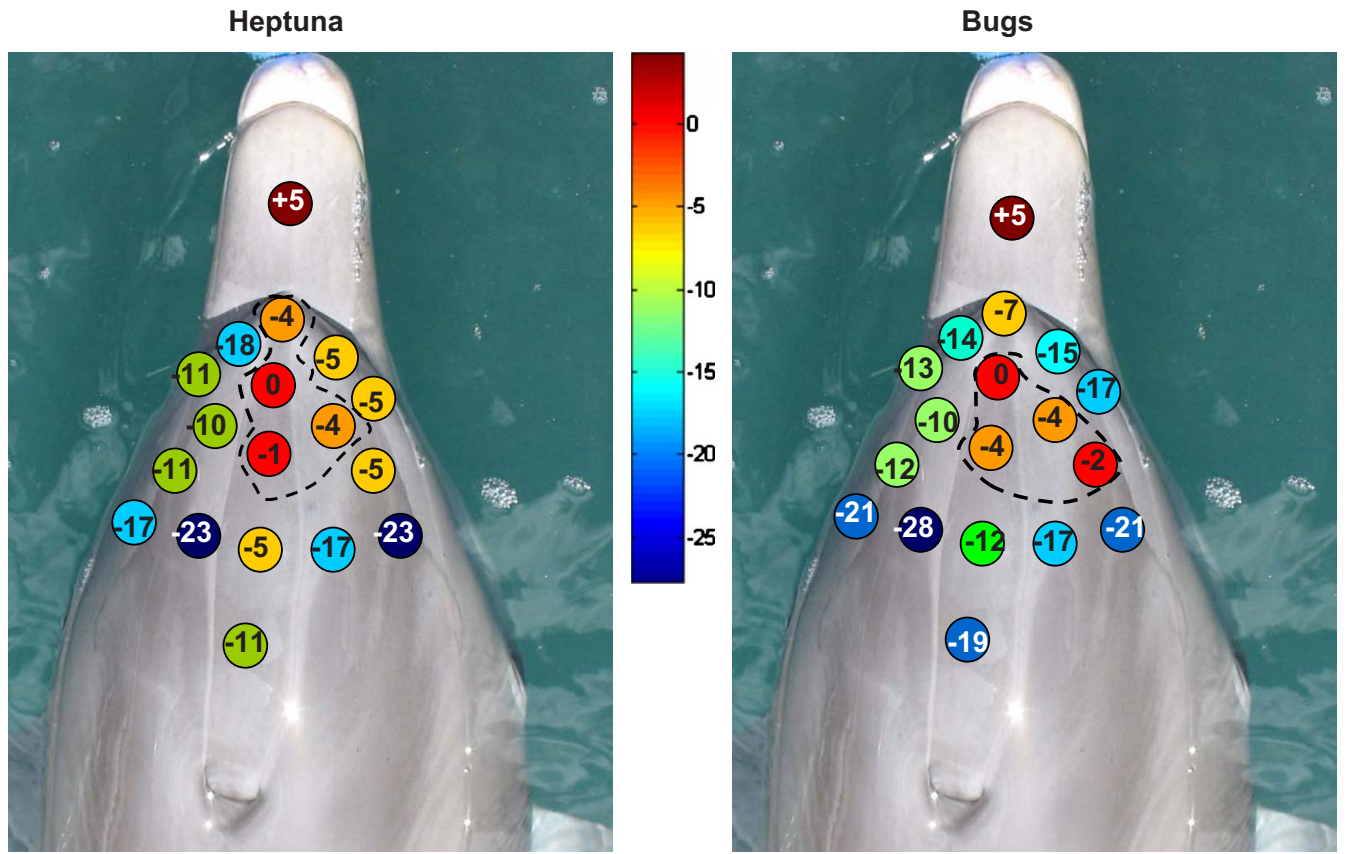

FIG. 9. (Color online) The relative amplitude distribution of the peak-to-peak amplitude on the forehead of the dolphins, reference to the hydrophone at location $\mathrm{C}$. The results obtained with the data from all the array geometries were used to create the relative amplitude map. The dashed line is an estimate of the area of maximum acoustic intensity on the animal's forehead when echolocating.

ence in the time delay between Heptuna and Bugs. The differences in the time delay between Heptuna and Bugs were probably caused by anatomical differences rather than inaccurate placement of the hydrophones. Although the forehead of a dolphin is very smooth with very few landmarks including skin coloration, the process of always having one hydrophone on the midline with one edge touching the rostrum insertion (location B) and a hydrophone at location $\mathrm{C}$ also on the midline with its edge touching the hydrophone at location B helped to minimize errors in hydrophone placement. Therefore, a single hydrophone could be repeatedly placed at the same location with an accuracy of approximately \pm 0.25 $\mathrm{cm}$.

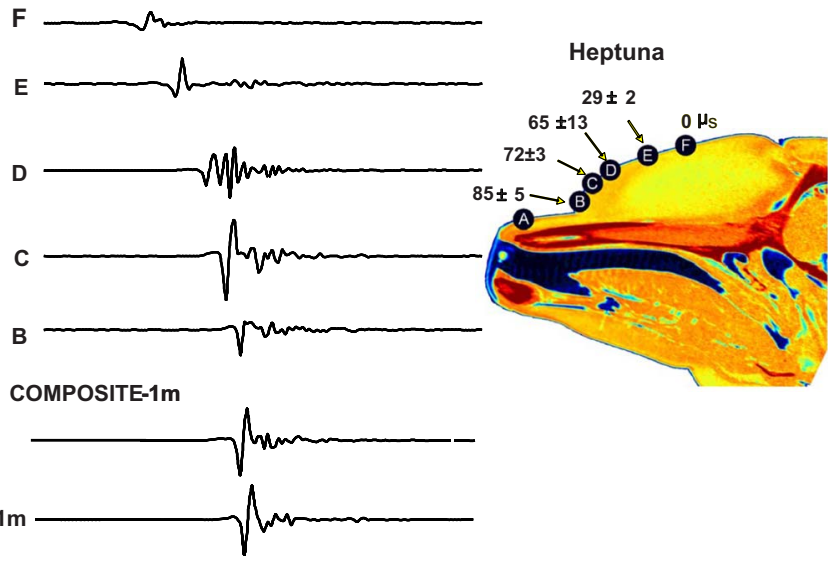

FIG. 10. (Color online) The waveform of an echolocation emission measured by the suction cup hydrophones in the linear array geometry for Heptuna and the summation of all the waveforms with the amplitude and time of occurrence of the signals are taken into account. The last waveform is that measured by the hydrophone at $1 \mathrm{~m}$. The mean and standard deviation of the time delays in receiving the signal at the various locations referenced to when the signal arrived at location $\mathrm{F}$ are also shown.
In both Figs. 10 and 11 are examples of a single echolocation signal measured by the various hydrophones on the animals' forehead. The time delay and amplitude of the signals were preserved in these two examples. Although the signals arrived at location $\mathrm{F}$ first before entering into the water the signals at location $\mathrm{F}$ have a longer propagation path to the hydrophone at $1 \mathrm{~m}$. The signals at locations $\mathrm{E}$ to $\mathrm{B}$ arrived in sequence and with a progressively shorter propagation distance in the water to the hydrophone at $1 \mathrm{~m}$. The signal that arrived at $1 \mathrm{~m}$ will be a summation of all the signals. In actuality, the signal in the far-field is the sum of the signal being radiated over the entire surface of the forehead. In order to gain an appreciation of the summation pro-

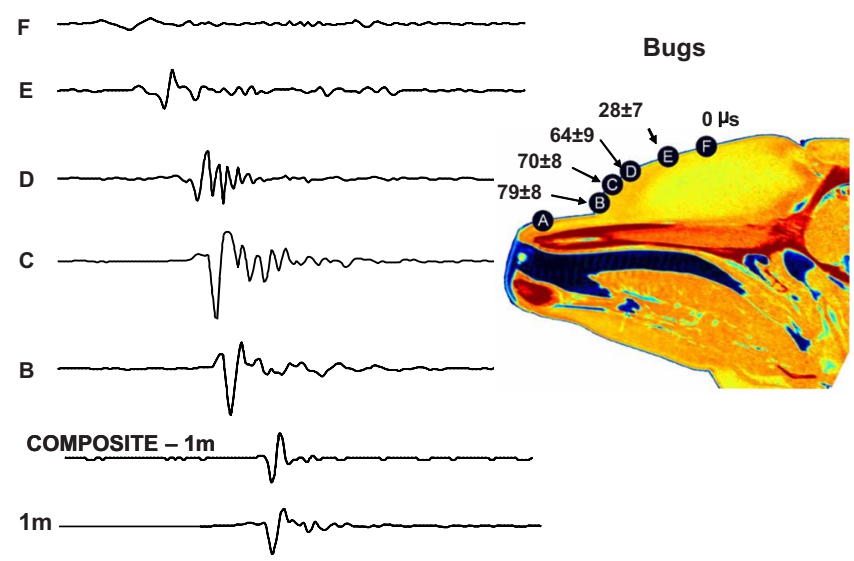

FIG. 11. (Color online) The waveform of an echolocation emission measured by the suction cup hydrophones in the linear array geometry for Bugs and the summation of all the waveforms with the amplitude and time of occurrence of the signals are taken into account. The last waveform is that measured by the hydrophone at $1 \mathrm{~m}$. The mean and standard deviation of the time delays in receiving the signal at the various locations referenced to when the signal arrived at location $\mathrm{F}$ are also shown. 
cess, the signal at each location was delayed relative to the signal measured at location $\mathrm{F}$ and then summed to produce the composite signal shown in both Figs. 10 and 11. This process can be visualize as having the signals measured at each location shifted toward the left by the amount of delay shown for each signal in the two figures. The important point is that the signal measured on the forehead appear distorted in relationship to the signal measured in the far-field at $1 \mathrm{~m}$. However, as the signal propagated into the far-field of the dolphin's head the composite signal resembled the signal measured at $1 \mathrm{~m}$. The signals measured at $1 \mathrm{~m}$ were typically 7-10 dB lower in amplitude than those measured at location C (see Fig. 3), and so its waveform along that of the composite signal was not plotted according to scale, otherwise the comparison between the composite and the far-field signal would be difficult to visualize.

\section{DISCUSSION AND CONCLUSIONS}

Section I was devoted mainly to the role of the melon in the focusing of acoustic signals generated by the phonic lips. However, the results obtained in this study especially with the hydrophones that were placed on the side of the forehead (see Fig. 9) suggest that the signals are first focused by the air sacs in the head and the focusing is refined by the sounds propagating through the melon. The geometry of the air sacs and their relative positions are particular difficult to described. Gurevich (1980) developed a mold cast technique, in which the shape of the air sacs in the nasal system of dolphins could be reproduced by injecting a casting material into the different nasal sacs (Ridgway, 1983). Nakamura et al. (1998) used RTV silicon and injected it into the air sacs of a common dolphin, Delphinus delphis. The silicon cast of Nakamura et al. (1998) is shown in Fig. 12(a). They did not inject the silicon into the left nasofrontal sac and the left nasofrontal sac in the figure is our estimate of what it would look like. The positions of the phonic lips were also estimated by Ridgway (personal communication). An example of one of Gurevich's casts for Tursiops truncates is shown in Fig. 12(b), while a schematic showing the relative position of important structures in the forehead of a Delphinis Delphis is shown in Fig. 12(c). Although the phonic lips are approximately 2-3 $\mathrm{cm}$ below the surface of the blowhole in the posterior portion of the forehead, our data show that most of the energy in echolocation clicks enter into the water at the anterior portion of the forehead, and very little energy is radiated from the sides and top of the forehead close to the location of the phonic lips. This characteristic strongly suggest that focusing by the airsacs and bony structures as well as diffraction within the melon is taking place. If we consider the photograph of Gurevich's cast taken from the perspective of the melon looking back toward the nasal system, one can imagine a parabolic dishlike reflective structure that would direct the acoustic energy forward rather than any other directions. However, the exact propagation pathways are not known. There is the distinct possibility that the role of the melon may be more of an impedance matching structure to efficiently allow sounds to enter into the water but yet have some focusing function. (a)

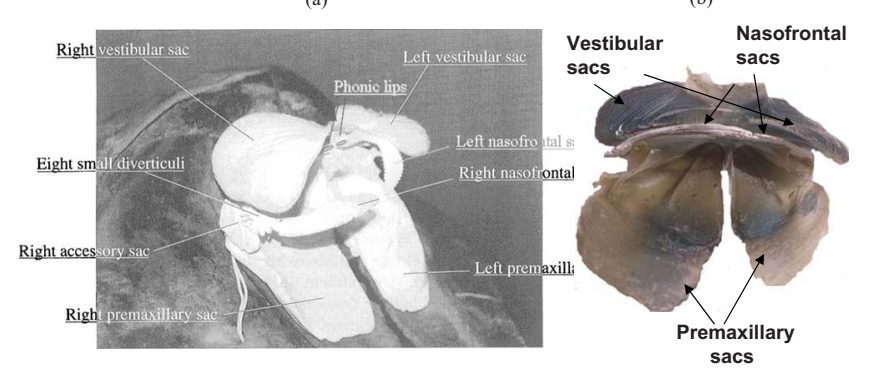

(c)

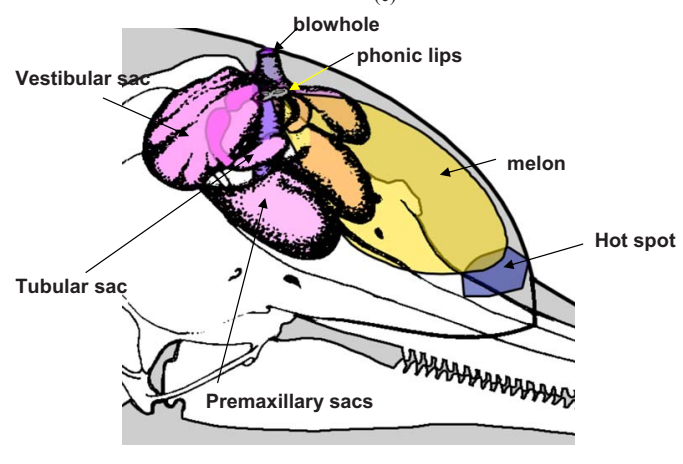

FIG. 12. (Color online) The silicon cast of Nakamura et al. (1998) is shown in panel (a) for a common dolphin. The left nasofrontal sac and phonic lips were drawn in by the authors. The cast in panel (b) was made by Vladimir Gurevich for a Tursiops truncates, and its picture was provided by Dr. Sam Ridgway. The perspective for Gurevich cast was from the front of the melon looking back toward the nasal system. The nasofrontal sacs were painted white. Panel (c) is a schematic showing the relative position of the air sacs and other internal structure of the forehead (adapted from Purves and Pilleri, 1983).

Most of our understanding of sound propagation within the head of dolphins comes from the numerical simulation work of Aroyan et al. (1992), which indicate that air sacs and bony structure are important to the transfer of energy from the phonic lips to the anterior portion of the forehead. Even the numerical simulation results of Aroyan does not convey the total picture. First of all, Aroyan technique assumed a continuous tonal source, and second, the CT scans were from deceased animals so that the shape and size of the different airsacs were approximated. With tonal signals, interferences from components of the signals reflecting off the various surfaces in the forehead will not cause a change in the frequency, as was found for the broadband signal (see Figs. 3, 10 , and 11).

The results from the linear array indicated that the region of maximum acoustic pressure on the surface of a dolphin's forehead was approximately $5.6 \mathrm{~cm}$ above the rostrum insertion, close to the midline of the forehead for Heptuna, and $4.2 \mathrm{~cm}$ above the rostrum insertion for Bugs. These two regions of maximum acoustic pressure was consistent between the two animals, and also consistent with the results from both linear-array measurements shown in Figs. 4 and 5. The small difference in the location of the maximum acoustic pressure could be attributed to anatomical differences in the forehead structure between the two dolphins. The point along the midline of the forehead, where the maximum acoustic pressure was measured, was directly forward of the low-density core measured by Norris and Harvey (1974). As with the study of Au et al. (2006) with harbor porpoises, the 
results of this study strongly support the hypothesis of Norris (1968) that sounds produced by the phonic lips propagate through the melon most effectively along the low-velocity core that is positioned almost in the middle of the melon. That the sounds are focused by the low-velocity core can be seen in the amplitude distribution graphs shown in Fig. 9. However, the results of this study also suggest that the air sacs may play a big role in the acoustic signals being projected toward the front of the animals rather than toward the sides and top of the animals forehead. The acoustic amplitudes dropped off quickly away from the midline, especially on the left side for both dolphins.

The asymmetry in the amplitude distribution on the dolphin's forehead can in part be attributed to the asymmetry in the size of the nares and the region that support the premaxillary sacs rest. The size of the port nares and the region supporting the premaxillary sacs are larger on the right side than the left side. Furthermore, the nares and premaxillary areas seem to be rotated slightly counter-clockwise (looking back from the tip of the rostrum) a few degrees. Nakamura $e t$ al. (1998) with their silicon reconstruction found that the left premaxillary sac had a smaller surface area than the right premaxillary sac. These anatomical differences can certainly affect how sounds propagate within the head to form the asymmetrical amplitude distribution on the forehead. The tilted orientation and difference in size of the premaxillary sacs could conceivably cause reflections off the top surface of the sacs to be directed toward the starboard side of the dolphin's head. Further research using numerical simulation of the kind performed by Aroyan et al., (1992) but with broadband signals is needed in order to resolve many of issues involved with sound propagation in the head of dolphins.

\section{ACKNOWLEDGMENT}

This work was supported by the U.S. Office of Naval Research, Mardi Hastings, program manager. Elizabeth Testa did the animal training/handling during this experiment. This study was conducted under the approval of the SPAWAR Institutional Animal Care and Use Committee (IACUC) and met the ASA standards for animal studies. We thank Dr. Sam Ridgway for his advise and for providing the photograph in Fig. 12(b). Thanks also goes to Alexis Rudd, who was responsible for creating Fig. 12(c). This is HIMB contribution No. 1385.

Aroyan, J. L., Cranford, T. W., Kent, J., and Norris, K. S. (1992). "Computer modeling of acoustic beam formation in Delphinus delphis," J. Acoust. Soc. Am. 92, 2539-2549.

$\mathrm{Au}, \mathrm{W}$. W. L. (1993). The Sonar of Dolphins (Springer Verlag, New York),
Au, W. W. L., Floyd, R. W., and Haun, J. E. (1978). "Propagation of dolphin echolocation signals," J. Acoust. Soc. Am. 64, 411-412.

Au, W. W. L., Kastelein, R. A., and Benoit-Bird, K. J. (2006). "Sound propagation in the head of an echolocating harbor porpoise (Phocoena phocoena)," J. Exp. Biol. 209, 2726-2733.

Au, W. W. L., Kastelein, R. A., Ripper, T., and Schooneman, N. M. (1999). "Transmission beam pattern and echolocation signals of a harbor porpoise (Phocoena phocoena)," J. Acoust. Soc. Am. 106, 3699-3705.

Blomberg, J. (1974). "Unusual lipids. II. Head oil of the north Atlantic pilot whale, Globicephala melaena," Lipids 9, 461-470.

Cranford, T. (1988). "The anatomy of acoustic structures in the spinner dolphin forehead as shown by X-ray computed tomography and computer graphics," in Animal Sonar: Processes and Performance, edited by P. E. Nachtigall and P. W. B. Moore, (Plenum, New York), p. 67.

Cranford, T. W. (2000). "In search of impulse sound sources in odontocetes," in Hearing by Whales and Dolphins, edited by W. Au, A. N. Popper, and R. R. Fay (Springer-Verlag, New York), pp. 109-156.

Cranford, T. W., Amudin, M., and Norris, K. S. (1996). "Functional morphology and homology in the odontocete nasal complex: Implications for sound generation," J. Morphol. 228, 223-285.

Diercks, K. J., Trochta, R. T., and Evans, W. E. (1973). "Delphinid sonar measurement and analysis," J. Acoust. Soc. Am. 54, 200-204.

Gellerman, L. W. (1933). "Chance orders of alternating stimuli in visual discrimination experiments," J. Genet. Psychol. 42, 206-208.

Gurevich, V. S. (1980). "A reconstructing technique for the nasal air sac of toothed whales," in Animal Sonar Systems, edited by R.-G. Busnel and J. F. Fish, (Plenum Press, New York), pp. 905-908.

Litchfield, C., Ackman, R. G., Sipos, J. C., and Eaton, C. A. (1971). "Isovaleroyl triglycerids from the blubber and melon oils of the beluga whale (Delphinaterus leucas)," Lipids 6, 674-681.

Litchfield, C., Karol, R., and Greenberg, A. J. (1979). "Compositional topography of melon lipids in the Atlantic bottlenose dolphin (Tursiops truncatus): Implications for echolocation," Mar. Biol. (Berlin) 52, 285-290.

Moore, P. W., Dankiewicz, L. A., and Houser, D. M. (2008). "Beamwidth control and angular target detection in an echolocating bottlenose dolphin (Tursiops truncatus)," J. Acoust. Soc. Am. 124, 3324-3332.

Nakamura, K., Yamada, T. K., and Shimazaki, K. (1998). "Measurements of the nasal sacs of individual common dolphin, Delphinus delphis, and Dall's porpoise, Phocoenoides dalli, by means of silicon reconstruction," Mamml. Studies 23, 119-122.

Norris, K. S. (1968). "The evolution of acoustic mechanisms in odontocete cetaceans," in Evolution and Environment, edited by E. T. Drake (Yale University Press, New Haven, CT), pp. 297-324.

Norris, K. S., and Harvey, G. W. (1974). "Sound transmission in the porpoise head," J. Acoust. Soc. Am. 56, 659-664.

Purves, P. E., and Pilleri, G. (1983). Echolocation in Whales and Dolphins (Academic Press, London).

Ridgway, S. H. (1983). "Dolphin hearing and sound production I health and illness," in Hearing and Other Senses: Presentations in Honor of E. G. Wever, edited by R. R. Fay and G. Gourevitch (The Amphora Press, Gronton, CT), pp. 247-296

Urick, R. J. (1983). Principles of Underwater Sound (McGraw-Hill, New York).

Varanasi, U., and Malin, D. C. (1971). "Unique lipies of he porpoise (Tursiops gilli): Differences in triacylglycerols and wax esters of acoustic (mandibular canal and melon) and bubble tissues," Biochim. Biophys. Acta 231, 415-418.

Varanasi, U., and Malin, D. C. (1972). "Triaclglyceros characteristics of porpoise acoustic tissues: Molecular structure of diisovaleroylglycerids," Science 176, 926-928.

Wood, F. G. (1964). Discussion. Marine BioAcoustics, Vol. 2, edited by W. Tavolga, Pergamon Press, Oxford, England, pp. 395-396. 